\title{
A WHITE NOISE APPROACH TO PHASE SPACE FEYNMAN PATH INTEGRALS
}

UDC 519.21

\author{
W. BOCK AND M. GROTHAUS
}

We dedicate this article to Anatolii Skorohod, Volodymyr Korolyuk and Igor Kovalenko

\begin{abstract}
The concepts of phase space Feynman integrals in White Noise Analysis are established. As an example the harmonic oscillator is treated. The approach perfectly reproduces the right physics. I.e., solutions to the Schrödinger equation are obtained and the canonical commutation relations are satisfied. The latter can be shown, since we not only construct the integral but rather the Feynman integrand and the corresponding generating functional.
\end{abstract}

\section{INTRODUCTION}

As an alternative approach to quantum mechanics Feynman introduced the concept of path integrals $(4,5,6]$, which was developed into an extremely useful tool in many branches of theoretical physics. In this article we develop the concepts for realizing Feynman integrals in phase space in the framework of White Noise Analysis. The phase space Feynman integral for a particle moving from $y_{0}$ at time 0 to $y$ at time $t$ under the potential $V$ is given by

$$
\begin{gathered}
\mathrm{N} \int_{x(0)=y_{0}, x(t)=y} \int \exp \left(\frac{i}{\hbar} \int_{0}^{t} p \dot{x}-\frac{p^{2}}{2}-V(x, p) d \tau\right) \prod_{0<\tau<t} d p(\tau) d x(\tau), \\
\hbar=\frac{h}{2 \pi} .
\end{gathered}
$$

Here $\hbar$ is Planck's constant, and the integral is thought of as being over all position paths with $x(0)=y_{0}$ and $x(t)=y$ and all momentum paths. The missing restriction on the momentum variable at time 0 and time $t$ is an immediate consequence of the Heisenberg uncertainty relation, i.e., the fact that one cannot measure momentum and space variable at the same time. The path integral to the phase space has several advantages. First, the semi-classical approximation can be validated easier in a phase space formulation

2010 Mathematics Subject Classification. Primary 60H40, 46F25.

Key words and phrases. White noise analysis, Feynman integrals, mathematical physics.

The authors would like to thank the organizing and programme committee of the MSTAII conference for an interesting an stimulating meeting. Wolfgang Bock wants especially thank to Yuri Kondratiev for the opportunity to give a talk on this topic at the conference. Furthermore, the authors would like to thank Florian Conrad, Anna Hoffmann, Tobias Kuna and Ludwig Streit for helpful discussions. The financial support from the DFG project GR 1809/9-1, which enabled the authors to join the conference, is thankfully acknowledged.

The paper is based on the talk presented at the International Conference "Modern Stochastics: Theory and Applications II" held on September 7-11, 2010 at Kyiv National Taras Shevchenko University and dedicated to three anniversaries of prominent Ukrainian scientists: Anatolii Skorokhod, Volodymyr Korolyuk and Igor Kovalenko. 
and second that quantum mechanics are founded on the phase space, i.e., every quantum mechanical observable can be expressed as a function of the space and momentum. A discussion about phase space path integrals can be found in the monograph [2] and in the references therein.

In the last fifty years there have been many approaches for giving a mathematically rigorous meaning to the phase space path integral by using, e.g., analytic continuation; see [13, 14] or Fresnel integrals [2, 1]. Here we choose a white noise approach. White Noise Analysis is a mathematical framework which offers generalizations of concepts from finite-dimensional analysis, like differential operators and Fourier transform to an infinite-dimensional setting. We give a brief introduction to White Noise Analysis in Section 2; for more details see [10, 3, 11, 21, 18. Of special importance in White Noise Analysis are spaces of generalized functions and their characterizations. In this article we choose the space of Hida distributions; see Section 2.

The idea of realizing Feynman integrals within the white noise framework goes back to [12. There the authors used exponentials of quadratic (generalized) functions in order to give meaning to the Feynman integral in configuration space representation

$$
\mathrm{N} \int_{x(0)=y_{0}, x(t)=y} \exp \left(\frac{i}{\hbar} S(x)\right) \prod_{0<\tau<t} d x(\tau), \quad \hbar=\frac{h}{2 \pi},
$$

with the classical action $S(x)=\int_{0}^{t} \frac{1}{2} m \dot{x}^{2}-V(x) d \tau$. We use these concepts of quadratic actions in White Noise Analysis, which were further developed in [8] to give rigorous meaning to the Feynman integrand

$$
\begin{aligned}
I_{V}= & \mathrm{N} \exp \left(\frac{i}{\hbar} \int_{0}^{t} p(\tau) \dot{x}(\tau)-\frac{p(\tau)^{2}}{2 m} d \tau+\frac{1}{2} \int_{0}^{t} \dot{x}(\tau)^{2}+p(\tau)^{2} d \tau\right) \\
& \times \cdot \exp \left(-\frac{i}{\hbar} \int_{0}^{t} V(x(\tau), p(\tau), \tau) d \tau\right) \cdot \delta(x(t)-y)
\end{aligned}
$$

as a Hida distribution. In this expression the sum of the first and the third integral in the exponential is the action $S(x, p)$, and the delta function (Donsker's delta) serves to pin trajectories to $y$ at time $t$. The second integral is introduced to simulate the Lebesgue integral by a local compensation of the fall-off of the Gaussian reference measure $\mu$. Furthermore, we use a Brownian motion starting with $y_{0}$ as a position variable and the momentum variable is modeled by white noise, i.e.,

$$
x(\tau)=y(0)+\sqrt{\frac{\hbar}{m}} B(\tau), \quad p(\tau)=\omega(\tau), \quad 0 \leq \tau \leq t .
$$

The construction is done in terms of the $T$-transform (infinite-dimensional version of the Fourier transform w.r.t. a Gaussian measure), which characterizes Hida distributions; see Theorem 2.4. At the same time, the $T$-transform of the constructed Feynman integrands provides us with their generating functional. Finally, using the generating functional, we can show that the generalized expectation (generating functional at zero) gives the Green's function to the corresponding Schrödinger equation. Moreover, with help of the generating functional we can show that the canonical commutation relations are fulfilled. This confirms on a mathematically rigorous level the heuristics developed in [6].

These are the core results of this article:

- The concepts of generalized Gauss kernels from 8 8 are extended to the vectorvalued case explicitly.

- The concepts for realizing the Feynman integrands in phase space in White Noise Analysis are provided. 
- The free Feynman integrand $I_{0}$ and the Feynman integrand for the harmonic oscillator $I_{H O}$ in phase space are constructed as Hida distributions; see Theorem 3.1 and Theorem 3.2 ,

- The results in Theorem 3.1 and Theorem 3.2 provide us with the generating functional to the Feynman integrands. The generalized expectations (generating functional at zero) provide us the Green's functions to the corresponding Schrödinger equation.

- In Theorem 4.4 the canonical commutator relations for $I_{0}$ are obtained in the sense of Feynman and Hibbs; see [6].

\section{White Noise Analysis}

2.1. Gel'fand triples. The starting point is the Gel'fand triple $S_{d}(\mathbb{R}) \subset L_{d}^{2}(\mathbb{R}, d x) \subset$ $S_{d}^{\prime}(\mathbb{R})$ of the $\mathbb{R}^{d}$-valued Schwartz test functions and tempered distributions with the Hilbert space of (equivalence classes of) $\mathbb{R}^{d}$-valued square integrable functions w.r.t. the Lebesgue measure as central space (equipped with its canonical inner product $(\cdot, \cdot)$ and norm $\|\cdot\|)$; for more details see e.g. [25, Exam. 11]. Since $S_{d}(\mathbb{R})$ is a nuclear space, represented as the projective limit of a decreasing chain of Hilbert spaces $\left(H_{p}\right)_{p \in \mathbb{N}}$ (see e.g. [23, Chap. 2] and [9]) i.e.,

$$
S_{d}(\mathbb{R})=\bigcap_{p \in \mathbb{N}} H_{p}
$$

we have that $S_{d}(\mathbb{R})$ is a countably Hilbert space in the sense of Gel'fand and Vilenkin [9]. We denote the inner product and the corresponding norm on $H_{p}$ by $(\cdot, \cdot)_{p}$ and $\|\cdot\|_{p}$, respectively, with the convention $H_{0}=L^{2}(\mathbb{R}, d x)$. Let $H_{-p}$ be the dual space of $H_{p}$ and let $\langle\cdot, \cdot\rangle$ denote the dual pairing on $H_{p} \times H_{-p} . H_{p}$ is continuously embedded into $L_{d}^{2}(\mathbb{R}, d x)$. By identifying $L_{d}^{2}(\mathbb{R}, d x)$ with its dual $L_{d}^{2}(\mathbb{R}, d x)^{\prime}$, via the Riesz isomorphism, we obtain the chain $H_{p} \subset L_{d}^{2}(\mathbb{R}, d x) \subset H_{-p}$. Note that $S_{d}^{\prime}(\mathbb{R})=\bigcup_{p \in \mathbb{N}} H_{-p}$, i.e., $S_{d}^{\prime}(\mathbb{R})$ is the inductive limit of the increasing chain of Hilbert spaces $\left(H_{-p}\right)_{p \in \mathbb{N}}$; see e.g. [9]. We denote the dual pairing of $S_{d}(\mathbb{R})$ and $S_{d}^{\prime}(\mathbb{R})$ also by $\langle\cdot, \cdot\rangle$. Note that its restriction on $S_{d}(\mathbb{R}) \times L_{d}^{2}(\mathbb{R}, d x)$ is given by $(\cdot, \cdot)$. We also use the complexifications of these spaces denoted with the subindex $\mathbb{C}$ (as well as their inner products and norms). The dual pairing we extend in a bilinear way. Hence we have the relation

$$
\langle g, f\rangle=(g, \bar{f}), \quad f, g \in L^{2}(\mathbb{R})_{\mathbb{C}},
$$

where the overline denotes the complex conjugation.

2.2. White noise spaces. We consider on $S_{d}^{\prime}(\mathbb{R})$ the $\sigma$-algebra $\mathcal{C}_{\sigma}\left(S_{d}^{\prime}(\mathbb{R})\right)$ generated by the cylinder sets $\left\{\omega \in S_{d}^{\prime}(\mathbb{R}) \mid\left\langle\xi_{1}, \omega\right\rangle \in F_{1}, \ldots,\left\langle\xi_{n}, \omega\right\rangle \in F_{n}\right\}, \xi_{i} \in S_{d}(\mathbb{R}), F_{i} \in \mathcal{B}(\mathbb{R})$, $1 \leq i \leq n, n \in \mathbb{N}$, where $\mathcal{B}(\mathbb{R})$ denotes the Borel $\sigma$-algebra on $\mathbb{R}$.

The canonical Gaussian measure $\mu$ on $C_{\sigma}\left(S_{d}^{\prime}(\mathbb{R})\right)$ is given via its characteristic function

$$
\int_{S_{d}^{\prime}(\mathbb{R})} \exp (i\langle\mathbf{f}, \omega\rangle) d \mu(\omega)=\exp \left(-\frac{1}{2}\|\mathbf{f}\|^{2}\right), \quad \mathbf{f} \in S_{d}(\mathbb{R}),
$$

by the theorem of Bochner and Minlos; see e.g. [20], [3, Chap. 2, Theo. 1. 11], and [11]. The space $\left(S_{d}^{\prime}(\mathbb{R}), \mathcal{C}_{\sigma}\left(S_{d}^{\prime}(\mathbb{R})\right), \mu\right)$ is the basic probability space in our setup. The central Gaussian spaces in our framework are the Hilbert spaces $\left(L^{2}\right):=L^{2}\left(S_{d}^{\prime}(\mathbb{R}), \mathcal{C}_{\sigma}\left(S_{d}^{\prime}(\mathbb{R})\right), \mu\right)$ of complex-valued square integrable functions w.r.t. the Gaussian measure $\mu$.

Within this formalism a version of a $d$-dimensional Brownian motion is given by

$$
\mathbf{B}(t, \boldsymbol{\omega}):=\left(\left\langle\mathbb{1}_{[0, t)}, \omega_{1}\right\rangle, \ldots\left\langle\mathbb{1}_{[0, t)}, \omega_{d}\right\rangle\right), \quad \boldsymbol{\omega}=\left(\omega_{1}, \ldots \omega_{d}\right) \in S_{d}^{\prime}(\mathbb{R}), t \geq 0,
$$

in the sense of an $\left(L^{2}\right)$-limit. Here $\mathbb{1}_{A}$ denotes the indicator function of a set $A$. 
2.3. The Hida triple. Let us now consider the complex Hilbert space $\left(L^{2}\right)$ and the corresponding Gel'fand triple

$$
(S) \subset\left(L^{2}\right) \subset(S)^{\prime} .
$$

Here $(S)$ denotes the space of Hida test functions and $(S)^{\prime}$ the space of Hida distributions. In the following we denote the dual pairing between elements of $(S)$ and $(S)^{\prime}$ by $\langle\langle\cdot, \cdot\rangle\rangle$. Instead of reproducing the construction of $(S)^{\prime}$ here we give its characterization in terms of the $T$-transform.

Definition 2.1. We define the $T$-transform of $\Phi \in(S)^{\prime}$ by

$$
T \Phi(\mathbf{f}):=\langle\langle\Phi, \exp (i\langle\mathbf{f}, \cdot\rangle)\rangle\rangle, \quad \mathbf{f}:=\left(f_{1}, \ldots, f_{d}\right) \in S_{d}(\mathbb{R}) .
$$

Remark 2.2. (i) Since $\exp (i\langle\mathbf{f}, \cdot\rangle) \in(S)$ for all $f \in S_{d}(\mathbb{R})$, the $T$-transform of a Hida distribution is well defined.

(ii) For $\mathbf{f}=0$ the above expression yields $\langle\langle\Phi, 1\rangle\rangle$, therefore, $T \Phi(0)$ is called the generalized expectation of $\Phi \in(S)^{\prime}$.

In order to characterize the space $(S)^{\prime}$ by the $T$-transform we need the following definition.

Definition 2.3. A mapping $F: S_{d}(\mathbb{R}) \rightarrow \mathbb{C}$ is called a $U$-functional if it satisfies the following conditions:

U1. For all $\mathbf{f}, \mathbf{g} \in S_{d}(\mathbb{R})$ the mapping $\mathbb{R} \ni \lambda \mapsto F(\lambda \mathbf{f}+\mathbf{g}) \in \mathbb{C}$ has an analytic continuation to $\lambda \in \mathbb{C}$ (ray analyticity).

U2. There exist constants $0<K, C<\infty$ and a $p \in \mathbb{N}_{0}$ such that

$$
|F(z \mathbf{f})| \leq K \exp \left(C|z|^{2}\|\mathbf{f}\|_{p}^{2}\right),
$$

for all $z \in \mathbb{C}$ and $\mathbf{f} \in S_{d}(\mathbb{R})$ (growth condition).

This is the basis of the following characterization theorem. For the proof we refer to [22, 17, 11, 16].

Theorem 2.4. A mapping $F: S_{d}(\mathbb{R}) \rightarrow \mathbb{C}$ is the $T$-transform of an element in $(S)^{\prime}$ if and only if it is a U-functional.

Theorem 2.4 enables us to discuss convergence of sequences of Hida distributions by considering the corresponding $T$-transforms, i.e., by considering convergence on the level of $U$-functionals. The following corollary is proved in [22, 11, 16].

Corollary 2.5. Let $\left(\Phi_{n}\right)_{n \in \mathbb{N}}$ denote a sequence in $(S)^{\prime}$ such that:

(i) For all $\mathbf{f} \in S_{d}(\mathbb{R}),\left(\left(T \Phi_{n}\right)(\mathbf{f})\right)_{n \in \mathbb{N}}$ is a Cauchy sequence in $\mathbb{C}$.

(ii) There exist constants $0<C, D<\infty$ such that for some $p \in \mathbb{N}_{0}$ one has

$$
\left|\left(T \Phi_{n}\right)(z \mathbf{f})\right| \leq D \exp \left(C|z|^{2}\|\mathbf{f}\|_{p}^{2}\right)
$$

for all $\mathbf{f} \in S_{d}(\mathbb{R}), z \in \mathbb{C}, n \in \mathbb{N}$.

Then $\left(\Phi_{n}\right)_{n \in \mathbb{N}}$ converges strongly in $(S)^{\prime}$ to a unique Hida distribution.

Example 2.6 (Vector-valued white noise). Let $\mathbf{B}(t), t \geq 0$ be the $d$-dimensional Brownian motion as in (4). Consider

$$
\frac{\mathbf{B}(t+h, \boldsymbol{\omega})-\mathbf{B}(t, \boldsymbol{\omega})}{h}=\left(\left\langle\frac{\mathbb{1}_{[t, t+h)}}{h}, \omega_{1}\right\rangle, \ldots,\left\langle\frac{\mathbb{1}_{[t, t+h)}}{h}, \omega_{d}\right\rangle\right), \quad h>0 .
$$

Then in the sense of Corollary 2.5 we have

$$
\left\langle\delta_{t}, \boldsymbol{\omega}\right\rangle:=\left(\left\langle\delta_{t}, \omega_{1}\right\rangle, \ldots,\left\langle\delta_{t}, \omega_{d}\right\rangle\right):=\lim _{h \searrow 0} \frac{\mathbf{B}(t+h, \boldsymbol{\omega})-\mathbf{B}(t, \boldsymbol{\omega})}{h} .
$$


Of course for the left derivative we get the same limit. Hence it is natural to call the generalized process $\left\langle\delta_{t}, \boldsymbol{\omega}\right\rangle, t \geq 0$, in $(S)^{\prime}$ vector-valued white noise. One also uses the notation $\boldsymbol{\omega}(t)=\left\langle\delta_{t}, \boldsymbol{\omega}\right\rangle, t \geq 0$.

Another useful corollary of Theorem 2.4 concerns integration of a family of generalized functions; see [22, 11, 16].

Corollary 2.7. Let $(\Lambda, \mathcal{A}, \nu)$ be a measure space and $\Lambda \ni \lambda \mapsto \Phi(\lambda) \in(S)^{\prime}$ a mapping. We assume that its $T$-transform $T \Phi$ satisfies the following conditions:

(i) The mapping $\lambda \mapsto T(\Phi(\lambda))(\mathbf{f})$ is measurable for all $\mathbf{f} \in S_{d}(\mathbb{R})$.

(ii) There exists a $p \in \mathbb{N}_{0}$ and functions $C \in L^{\infty}(\Lambda, \nu)$ and $D \in L^{1}(\Lambda, \nu)$ such that

$$
|T(\Phi(\lambda))(z \mathbf{f})| \leq D(\lambda) \exp \left(C(\lambda)|z|^{2}\|\mathbf{f}\|^{2}\right),
$$

for a.e. $\lambda \in \Lambda$ and for all $\mathbf{f} \in S_{d}(\mathbb{R}), z \in \mathbb{C}$.

Then, in the sense of Bochner integration in $H_{-q} \subset(S)^{\prime}$ for a suitable $q \in \mathbb{N}_{0}$, the integral of the family of Hida distributions is itself a Hida distribution, i.e., $\int_{\Lambda} \Phi(\lambda) d \nu(\lambda) \in(S)^{\prime}$ and the T-transform interchanges with integration, i.e.,

$$
T\left(\int_{\Lambda} \Phi(\lambda) d \nu(\lambda)\right)=\int_{\Lambda} T(\Phi(\lambda)) d \nu(\lambda)
$$

Based on the above theorem, we introduce the following Hida distribution.

Definition 2.8. We define Donsker's delta at $x \in \mathbb{R}$ corresponding to $0 \neq \boldsymbol{\eta} \in L_{d}^{2}(\mathbb{R})$ by

$$
\delta_{x}(\langle\boldsymbol{\eta}, \cdot\rangle):=\frac{1}{2 \pi} \int_{\mathbb{R}} \exp (i \lambda(\langle\boldsymbol{\eta}, \cdot\rangle-x)) d \lambda
$$

in the sense of Bochner integration; see e.g. [11, 19, 25]. Its $T$-transform in $\mathbf{f} \in S_{d}(\mathbb{R})$ is given by

$$
T\left(\delta_{x}(\langle\boldsymbol{\eta}, \cdot\rangle)(\mathbf{f})=\frac{1}{\sqrt{2 \pi\langle\boldsymbol{\eta}, \boldsymbol{\eta}\rangle}} \exp \left(-\frac{1}{2\langle\boldsymbol{\eta}, \boldsymbol{\eta}\rangle}(i\langle\boldsymbol{\eta}, \mathbf{f}\rangle-x)^{2}-\frac{1}{2}\langle\mathbf{f}, \mathbf{f}\rangle\right) .\right.
$$

2.4. Generalized Gauss kernels. Here we review a special class of Hida distributions which are defined by their $T$-transform; see e.g. [8]. Let $\mathcal{B}$ be the set of all continuous bilinear mappings $B: S_{d}(\mathbb{R}) \times S_{d}(\mathbb{R}) \rightarrow \mathbb{C}$. Then the functions

$$
S_{d}(\mathbb{R}) \ni f \mapsto \exp \left(-\frac{1}{2} B(\mathbf{f}, \mathbf{f})\right) \in \mathbb{C}
$$

for all $B \in \mathcal{B}$ are $U$-functionals. Therefore, by using the characterization of Hida distributions in Theorem 2.4, the inverse $T$-transform of the functions

$$
\Phi_{B}:=T^{-1} \exp \left(-\frac{1}{2} B\right)
$$

are elements of $(S)^{\prime}$.

Definition 2.9. The set of generalized Gauss kernels is defined by

$$
G G K:=\left\{\Phi_{B}, B \in \mathcal{B}\right\} .
$$

Example 2.10 ([8]). We consider a symmetric trace class operator $K$ on $L_{d}^{2}(\mathbb{R})$ such that $-\frac{1}{2}<K \leq 0$, then

$$
\int_{S_{d}^{\prime}(\mathbb{R})} \exp (-\langle\omega, K \omega\rangle) d \mu(\omega)=(\operatorname{det}(I d+2 K))^{-1 / 2}<\infty .
$$

For the definition of $\langle\cdot, K \cdot\rangle$ see the remark below. Here $I d$ denotes the identity operator on the Hilbert space $L_{d}^{2}(\mathbb{R})$, and $\operatorname{det}(A)$ of a symmetric trace class operator $A$ on $L_{d}^{2}(\mathbb{R})$ 
denotes the infinite product of its eigenvalues, if it exists. In the present situation we have $\operatorname{det}(I d+2 K) \neq 0$. Therefore we obtain that the exponential $g=\exp \left(-\frac{1}{2}\langle\cdot, K \cdot\rangle\right)$ is square-integrable and its $T$-transform is given by

$$
T g(\mathbf{f})=(\operatorname{det}(I d+K))^{-1 / 2} \exp \left(-\frac{1}{2}\left(\mathbf{f},(I d+K)^{-1} \mathbf{f}\right)\right), \quad \mathbf{f} \in S_{d}(\mathbb{R}) .
$$

Therefore, $(\operatorname{det}(I d+K))^{1 / 2} g$ is a generalized Gauss kernel.

Remark 2.11. Since a trace class operator is compact (see e.g. [23]), we have that $K$ in the above example is diagonalizable, i.e.,

$$
K f=\sum_{k=1}^{\infty} k_{n}\left(f, e_{n}\right) e_{n}, \quad f \in L^{2}(\mathbb{R}, d x),
$$

where $\left(e_{n}\right)_{n \in \mathbb{N}}$ denotes an eigenbasis of the corresponding eigenvalues $\left(k_{n}\right)_{n \in \mathbb{N}}$ with $k_{n} \in\left(-\frac{1}{2}, 0\right]$, for all $n \in \mathbb{N}$. Since $K$ is compact, we have that $\lim _{n \rightarrow \infty} k_{n}=0$ and since $K$ is trace class we also have $\sum_{n=1}^{\infty}\left(e_{n},-K e_{n}\right)<\infty$. We define for $\omega \in S_{d}^{\prime}(\mathbb{R})$,

$$
-\langle\omega, K \omega\rangle:=\lim _{N \rightarrow \infty} \sum_{n=1}^{N}\left\langle e_{n}, \omega\right\rangle\left(-k_{n}\right)\left\langle e_{n}, \omega\right\rangle .
$$

Then as a limit of measurable functions $\omega \mapsto-\langle\omega, K \omega\rangle$ is measurable and hence

$$
\int_{S_{d}^{\prime}(\mathbb{R})} \exp (-\langle\omega, K \omega\rangle) d \mu(\omega) \in[0, \infty]
$$

The explicit formula for the $T$-transform and expectation then follow by a straightforward calculation with help of the above limit procedure.

Definition 2.12. Let $K: L_{d, \mathbb{C}}^{2}(\mathbb{R}, d x) \rightarrow L_{d, \mathbb{C}}^{2}(\mathbb{R}, d x)$ be linear and continuous such that

(i) $I d+K$ is injective,

(ii) there exists $p \in \mathbb{N}_{0}$ such that $(I d+K)\left(L_{d, \mathbb{C}}^{2}(\mathbb{R}, d x)\right) \subset H_{p, \mathbb{C}}$ is dense,

(iii) there exist $q \in \mathbb{N}_{0}$ such that $(I d+K)^{-1}: H_{p, \mathbb{C}} \rightarrow H_{-q, \mathbb{C}}$ is continuous with $p$ as in (ii).

Then we define the normalized exponential

$$
\mathrm{N} \exp \left(-\frac{1}{2}\langle\cdot, K \cdot\rangle\right)
$$

by

$$
T\left(\mathrm{~N} \exp \left(-\frac{1}{2}\langle\cdot, K \cdot\rangle\right)\right)(\mathbf{f}):=\exp \left(-\frac{1}{2}\left\langle\mathbf{f},(I d+K)^{-1} \mathbf{f}\right\rangle\right), \quad \mathbf{f} \in S_{d}(\mathbb{R})
$$

Remark 2.13. The "normalization" of the exponential in the above definition can be regarded as a division of a divergent factor. In an informal way one can write

$$
T\left(\mathrm{~N} \exp \left(-\frac{1}{2}\langle\cdot, K \cdot\rangle\right)\right)(\mathbf{f})=\frac{T\left(\exp \left(-\frac{1}{2}\langle\cdot, K \cdot\rangle\right)\right)(f)}{T\left(\exp \left(-\frac{1}{2}\langle\cdot, K \cdot\rangle\right)\right)(0)}=\frac{T\left(\exp \left(-\frac{1}{2}\langle\cdot, K \cdot\rangle\right)\right)(f)}{\sqrt{\operatorname{det}(I d+K)}},
$$

i.e., if the determinant in Example 2.10 above is not defined, we can still define the normalized exponential by the $T$-transform without the diverging prefactor. The assumptions in the above definition then guarantee the existence of the generalized Gauss kernel in (5). 
Example 2.14. For sufficiently "nice" operators $K$ and $L$ on $L_{d}^{2}(\mathbb{R})_{\mathbb{C}}$ we can define the product

$$
\mathrm{N} \exp \left(-\frac{1}{2}\langle\cdot, K \cdot\rangle\right) \cdot \exp \left(-\frac{1}{2}\langle\cdot, L \cdot\rangle\right)
$$

of two square-integrable functions. Its $T$-transform is then given by

$$
\begin{aligned}
& T\left(\mathrm{~N} \exp \left(-\frac{1}{2}\langle\cdot, K \cdot\rangle\right) \cdot \exp \left(-\frac{1}{2}\langle\cdot, L \cdot\rangle\right)\right)(\mathbf{f}) \\
& \quad=\sqrt{\frac{1}{\operatorname{det}\left(I d+L(I d+K)^{-1}\right)}} \exp \left(-\frac{1}{2}\left\langle\mathbf{f},(I d+K+L)^{-1} \mathbf{f}\right\rangle\right), \quad \mathbf{f} \in S_{d}(\mathbb{R}),
\end{aligned}
$$

in this case the left-hand side is indeed a $U$-functional.

In the case $g \in S_{d}(\mathbb{R}), c \in \mathbb{C}$ the product between the Hida distribution $\Phi$ and the Hida test function $\exp (i\langle g, \cdot\rangle+c)$ is well defined because $(S)$ is a continuous algebra under pointwise multiplication. The next definition is an extension of this product.

Definition 2.15. The pointwise product of a Hida distribution $\Phi \in(S)^{\prime}$ with an exponential of a linear term, i.e.,

$$
\Phi \cdot \exp (i\langle\mathbf{g}, \cdot\rangle+c), \quad \mathbf{g} \in L_{d}^{2}(\mathbb{R})_{\mathbb{C}}, c \in \mathbb{C},
$$

is defined by

$$
T(\Phi \cdot \exp (i\langle\mathbf{g}, \cdot\rangle+c))(\mathbf{f}):=T \Phi(\mathbf{f}+\mathbf{g}) \exp (c), \quad \mathbf{f} \in S_{d}(\mathbb{R}),
$$

if $T \Phi$ has a continuous extension to $L_{d}^{2}(\mathbb{R})_{\mathbb{C}}$ and the term on the right-hand side is a $U$-functional in $\mathbf{f} \in S_{d}(\mathbb{R})$.

Definition 2.16. Let $D \subset \mathbb{R}$ such, that $0 \in \bar{D}$. Under the assumption that $T \Phi$ has a continuous extension to $L_{d}^{2}(\mathbb{R})_{\mathbb{C}}, \boldsymbol{\eta} \in L_{d}^{2}(\mathbb{R})_{\mathbb{C}}, y \in \mathbb{R}, \lambda \in \gamma_{\alpha}:=\{\exp (-i \alpha) s \mid s \in \mathbb{R}\}$ and that the integrand

$$
\gamma_{\alpha} \ni \lambda \mapsto \exp (-i \lambda y) T \Phi(\mathbf{f}+\lambda \boldsymbol{\eta}) \in \mathbb{C}
$$

fulfills the conditions of Corollary 2.7 for all $\alpha \in D$, one can define the product

$$
\Phi \cdot \delta(\langle\boldsymbol{\eta}, \cdot\rangle-y)
$$

by

$$
T(\Phi \cdot \delta(\langle\boldsymbol{\eta}, \cdot\rangle-y))(\mathbf{f}):=\lim _{\alpha \rightarrow 0} \int_{\gamma_{\alpha}} \exp (-i \lambda y) T \Phi(\mathbf{f}+\lambda \boldsymbol{\eta}) d \lambda,
$$

of course, under the assumption that the right-hand side converges in the sense of Corollary 2.5, see e.g. 8].

Lemma 2.17. Let $L$ be a $d \times d$ block operator matrix on $L_{d}^{2}(\mathbb{R})_{\mathbb{C}}$ acting componentwise such that all entries are bounded operators on $L^{2}(\mathbb{R})_{\mathbb{C}}$. Let $K$ be a $d \times d$ block operator matrix on $L_{d}^{2}(\mathbb{R})_{\mathbb{C}}$, such that $I d+K$ and $N=I d+K+L$ are bounded with bounded inverse. Furthermore, assume that $\operatorname{det}\left(I d+L(I d+K)^{-1}\right)$ exists and is different from zero (this is, e.g., the case if $L$ is trace class and -1 in the resolvent set of $L(I d+K)^{-1}$ ). Let $M_{N^{-1}}$ be the matrix given by an orthogonal system $\left(\boldsymbol{\eta}_{k}\right)_{k=1, \ldots J}$ of nonzero functions from $L_{d}^{2}(\mathbb{R}), J \in \mathbb{N}$, under the bilinear form $\left(\cdot, N^{-1} \cdot\right)$, i.e., $\left(M_{N^{-1}}\right)_{i, j}=\left(\boldsymbol{\eta}_{i}, N^{-1} \boldsymbol{\eta}_{j}\right)$. Under the assumption that either

$$
\Re\left(M_{N^{-1}}\right)>0 \quad \text { or } \quad \Re\left(M_{N^{-1}}\right)=0 \text { and } \Im\left(M_{N^{-1}}\right) \neq 0,
$$

where $M_{N^{-1}}=\Re\left(M_{N^{-1}}\right)+i \Im\left(M_{N^{-1}}\right)$ with real matrices $\Re\left(M_{N^{-1}}\right)$ and $\Im\left(M_{N^{-1}}\right)$, then

$$
\Phi_{K, L}:=\mathrm{N} \exp \left(-\frac{1}{2}\langle\cdot, K \cdot\rangle\right) \cdot \exp \left(-\frac{1}{2}\langle\cdot, L \cdot\rangle\right) \cdot \exp (i\langle\cdot, \mathbf{g}\rangle) \cdot \prod_{i=1}^{J} \delta\left(\left\langle\cdot, \boldsymbol{\eta}_{k}\right\rangle-y_{k}\right),
$$


for $\mathbf{g} \in L_{d}^{2}(\mathbb{R}, \mathbb{C}), t>0, y_{k} \in \mathbb{R}, k=1, \ldots, J$, exists as a Hida distribution. Moreover, for $\mathbf{f} \in S_{d}(\mathbb{R})$,

$$
\begin{aligned}
T \Phi_{K, L}(\mathbf{f})= & \frac{1}{\sqrt{(2 \pi)^{J} \operatorname{det}\left(\left(M_{N^{-1}}\right)\right)}} \sqrt{\frac{1}{\operatorname{det}\left(I d+L(I d+K)^{-1}\right)}} \\
& \times \exp \left(-\frac{1}{2}\left((\mathbf{f}+\mathbf{g}), N^{-1}(\mathbf{f}+\mathbf{g})\right)\right) \exp \left(-\frac{1}{2}\left(u,\left(M_{N^{-1}}\right)^{-1} u\right)\right)
\end{aligned}
$$

where

$$
u=\left(\left(i y_{1}+\left(\boldsymbol{\eta}_{1}, N^{-1}(\mathbf{f}+\mathbf{g})\right)\right), \ldots,\left(i y_{J}+\left(\boldsymbol{\eta}_{J}, N^{-1}(\mathbf{f}+\mathbf{g})\right)\right)\right) .
$$

Proof. We want to give meaning to the expression

$$
\mathrm{N} \exp \left(-\frac{1}{2}\langle\cdot, K \cdot\rangle\right) \cdot \exp \left(-\frac{1}{2}\langle\cdot, L \cdot\rangle\right) \cdot \exp (i\langle\cdot, \mathbf{g}\rangle) \cdot \prod_{k=1}^{J} \delta\left(\left\langle\cdot, \boldsymbol{\eta}_{k}\right\rangle-y_{k}\right)
$$

using Definition 2.16 inductively. Note that

$$
\mathrm{N} \exp \left(-\frac{1}{2}\langle\cdot, K \cdot\rangle\right) \cdot \exp \left(-\frac{1}{2}\langle\cdot, L \cdot\rangle\right)
$$

can be defined as in Example 2.14. Hence we obtain for the T-transform of the integrand

$$
\begin{aligned}
\gamma_{\alpha}^{J} \ni \lambda \mapsto \Phi_{\lambda}= & \exp \left(-i \sum_{j=1}^{J} \lambda_{j} e^{-i \alpha} y_{j}\right) \cdot \exp \left(i \sum_{j=1}^{J} \lambda_{j} e^{-i \alpha}\left\langle\boldsymbol{\eta}_{j}, \cdot\right\rangle\right) \\
& \times \mathrm{N} \exp \left(-\frac{1}{2}\langle\cdot, K \cdot\rangle\right) \exp \left(-\frac{1}{2}\langle\cdot, L \cdot\rangle\right) \exp (i\langle\cdot, \mathbf{g}\rangle)
\end{aligned}
$$

in $\mathbf{f} \in S_{d}(\mathbb{R})$,

$$
\begin{aligned}
T(\exp ( & \left.-i \sum_{j=1}^{J} \lambda_{j} e^{-i \alpha} y_{j}\right) \exp \left(i \sum_{j=1}^{J} \lambda_{j} e^{-i \alpha}\left\langle\boldsymbol{\eta}_{j}, \cdot\right\rangle\right) \\
& \left.\times \mathrm{N} \exp \left(-\frac{1}{2}\langle\cdot, K \cdot\rangle\right) \exp \left(-\frac{1}{2}\langle\cdot, L \cdot\rangle\right) \exp (i\langle\cdot, \mathbf{g}\rangle)\right)(\mathbf{f}) \\
= & \exp \left(-i \sum_{j=1}^{J} \lambda_{j} e^{-i \alpha} y_{j}\right) \\
& \times T\left(\mathrm{~N} \exp \left(-\frac{1}{2}\langle\cdot, K \cdot\rangle\right) \exp \left(-\frac{1}{2}\langle\cdot, L \cdot\rangle\right) \exp \left(i\langle\cdot, \mathbf{g}\rangle+\sum_{j=1}^{J} \lambda_{j} e^{-i \alpha}\left\langle\boldsymbol{\eta}_{j}\right\rangle\right)\right) \\
= & \exp \left(-i \sum_{j=1}^{J} \lambda_{j} e^{-i \alpha} y_{j}\right) \frac{1}{\sqrt{\operatorname{det}\left(I d+L(I d+K)^{-1}\right)}} \\
& \times \exp \left(-\frac{1}{2}\left(\left(\mathbf{f}+\mathbf{g}+\sum_{j=1}^{J} \lambda_{j} e^{-i \alpha} \boldsymbol{\eta}_{j}\right), N^{-1}\left(\mathbf{f}+\mathbf{g}+\sum_{k=1}^{J} \lambda_{k} e^{-i \alpha} \boldsymbol{\eta}_{k}\right)\right)\right)
\end{aligned}
$$


Here we use $y=\left(y_{1}, \ldots, y_{J}\right)$ and $\lambda=\left(\lambda_{1}, \ldots, \lambda_{J}\right)$, respectively. Then we can rewrite the above formula with the help of the matrix $M_{N^{-1}}$ as

$$
\begin{aligned}
\gamma_{\alpha}^{J} \ni \lambda \mapsto T( & \exp \left(-i \sum_{j=1}^{J} \lambda_{j} e^{-i \alpha} y_{j}\right) \exp \left(i \sum_{j=1}^{J} \lambda_{j} e^{-i \alpha}\left\langle\boldsymbol{\eta}_{j}, \cdot\right\rangle\right) \\
& \left.\quad \times \operatorname{Nexp}\left(-\frac{1}{2}\langle\cdot, K \cdot\rangle\right) \exp \left(-\frac{1}{2}\langle\cdot, L \cdot\rangle\right) \exp (i\langle\cdot, \mathbf{g}\rangle)\right)(\mathbf{f}) \\
= & \frac{1}{\sqrt{\operatorname{det}\left(I d+L(I d+K)^{-1}\right)}} \\
& \times \exp \left(-\frac{1}{2} e^{-2 i \alpha}\left(\lambda, M_{N^{-1}} \lambda\right)\right. \\
& \left.\left.\quad-e^{-i \alpha} \lambda\left(\left((\mathbf{f}+\mathbf{g}), N^{-1} \boldsymbol{\eta}_{1}\right), \ldots\left((\mathbf{f}+\mathbf{g}), N^{-1} \boldsymbol{\eta}_{d}\right)\right)-i y\right)\right) .
\end{aligned}
$$

The function in (6) is integrable w.r.t. the Lebesgue measure, if the real part of $e^{-2 i \alpha} M_{N^{-1}}$, i.e., $\Re\left(e^{-2 i \alpha} M_{N^{-1}}\right)=\cos (2 \alpha) \Re\left(M_{N^{-1}}\right)+\sin (2 \alpha) \Im\left(M_{N^{-1}}\right)$, is positive definite. Our assumptions on $M_{N^{-1}}$ in Lemma 2.17 imply that this holds for $\alpha$ in a set $D$, as required in Definition 2.16. The calculation of the T-transform then follows in an analogous way to the calculation of the $T$-transform of a product of Donskers delta functions; see e.g. [19, 25].

\section{Phase space Feynman path integrals}

In the following we realize rigorously the ansatz

$$
\begin{aligned}
I_{V}= & \mathrm{N} \exp \left(\frac{i}{\hbar} \int_{0}^{t} p(\tau) \dot{x}(\tau)-\frac{p(\tau)^{2}}{2 m} d \tau+\frac{1}{2} \int_{0}^{t} \dot{x}(\tau)^{2}+p(\tau)^{2} d \tau\right) \\
& \times \exp \left(-\frac{i}{\hbar} \int_{0}^{t} V(x(\tau), p(\tau), \tau) d \tau\right) \cdot \delta(x(t)-y),
\end{aligned}
$$

for the Feynman integrand in phase space for $V=0$ (free particle) and the harmonic oscillator, i.e., $x \mapsto V(x)=\frac{1}{2} k x^{2}, k \geq 0$, motivated in the introduction; see (10).

3.1. The free Feynman integrand in phase space. First we consider $V=0$ (free particle). For simplicity let $\hbar=m=1$ and $y_{0}=0$. Furthermore, we choose to have one space dimension and one dimension for the corresponding momentum variable, i.e., the underlying space is $S_{2}(\mathbb{R})$. Note that the first term in (7) can be considered as an exponential of a quadratic type:

$$
\begin{aligned}
& \mathrm{N} \exp \left(i \int_{0}^{t}\left(p(\tau) \dot{x}(\tau)-\frac{p(\tau)^{2}}{2}\right) d \tau+\frac{1}{2} \int_{0}^{t} \dot{x}(\tau)^{2}+p(\tau)^{2} d \tau\right) \\
& =\operatorname{Nexp}\left(-\frac{1}{2}\left\langle\left(\omega_{x}, \omega_{p}\right), K\left(\omega_{x}, \omega_{p}\right)\right\rangle\right),
\end{aligned}
$$

where the operator matrix $K$ on $L_{2}^{2}(\mathbb{R})_{\mathbb{C}}$ can be written as

$$
K=\left(\begin{array}{cc}
-\mathbb{1}_{[0, t)} & -i \mathbb{1}_{[0, t)} \\
-i \mathbb{1}_{[0, t)} & -(1-i) \mathbb{1}_{[0, t)}
\end{array}\right) .
$$


Here the operator $\mathbb{1}_{[0, t)}$ denotes the multiplication with $\mathbb{1}_{[0, t)}$. Hence, the integrand in (7) can then be written as

$$
I_{0}=\mathrm{N} \exp \left(-\frac{1}{2}\left\langle\left(\omega_{x}, \omega_{p}\right), K\left(\omega_{x}, \omega_{p}\right)\right\rangle\right) \cdot \delta\left(\left\langle\left(\omega_{x}, \omega_{p}\right),\left(\mathbb{1}_{[0, t)}, 0\right)\right\rangle-y\right),
$$

where the last term pins the position variable to $y$ at $t$. Note that the momentum variable is not pinned. Our aim is to apply Lemma 2.17 with $K$ as above and $\mathbf{g}=0, L=0$ and as $\boldsymbol{\eta}=\left(\mathbb{1}_{[0, t)}, 0\right)$. The inverse of $(I d+K)$ is given by

$$
N^{-1}=(I d+K)^{-1}=\left(\begin{array}{cc}
\mathbb{1}_{[0, t)^{c}}+i \mathbb{1}_{[0, t)} & i \mathbb{1}_{[0, t)} \\
i \mathbb{1}_{[0, t)} & \mathbb{1}_{[0, t)^{c}}
\end{array}\right),
$$

hence $\left(\boldsymbol{\eta}, N^{-1} \boldsymbol{\eta}\right)=i \cdot t$. Therefore, the assumptions of Lemma 2.17 are fulfilled. Thus $I_{0}$ exists as a Hida distribution. By applying Lemma 2.17 its $T$-transform in $\left(f_{x}, f_{p}\right) \in S_{2}(\mathbb{R})$ is given by

$$
\begin{aligned}
T(\mathrm{~N} & \left.\exp \left(-\frac{1}{2}\left\langle\left(\omega_{x}, \omega_{p}\right), K\left(\omega_{x}, \omega_{p}\right)\right\rangle\right) \cdot \delta\left(\left\langle\left(\omega_{x}, \omega_{p}\right),\left(\mathbb{1}_{[0, t)}, 0\right)\right\rangle-y\right)\right)\left(f_{x}, f_{p}\right) \\
= & \frac{1}{\sqrt{2 \pi i t}} \exp \left(-\frac{1}{2 i t}\left(y-\int_{0}^{t} f_{x}+f_{p} d s\right)^{2}-\frac{1}{2}\left(\left(f_{x}, f_{p}\right), N^{-1}\left(f_{x}, f_{p}\right)\right)\right) \\
= & \frac{1}{\sqrt{2 \pi i t}} \exp \left(-\frac{1}{2 i t}\left(y-\int_{0}^{t} f_{x}+f_{p} d s\right)^{2}\right) \\
& \times \exp \left(-\frac{1}{2}\left(\left(f_{x}, f_{p}\right),\left(\begin{array}{c}
\mathbb{1}_{[0, t)^{c}}+i \mathbb{1}_{[0, t)} \\
i \mathbb{1}_{[0, t)}
\end{array} \mathbb{\mathbb { 1 }}_{[0, t)} \mathbb{1}_{[0, t)^{c}}\right)\left(f_{x}, f_{p}\right)\right)\right) \\
= & \frac{1}{\sqrt{2 \pi i t}} \exp \left(-\frac{1}{2 i t}\left(y-\int_{0}^{t} f_{x}+f_{p} d s\right)^{2}\right) \\
& \times \exp \left(-\frac{1}{2}\left(\int_{[0, t)^{c}} f_{x}^{2}+f_{p}^{2} d s+i \int_{[0, t)} f_{x}^{2} d s+2 i \int_{[0, t)} f_{x}(s) f_{p}(s) d s\right)\right) .
\end{aligned}
$$

Hence its generalized expectation

$$
\mathbb{E}\left(I_{0}\right)=T I_{0}(0)=\frac{1}{\sqrt{2 \pi i t}} \exp \left(-\frac{1}{2 i t} y^{2}\right)=K(y, t, 0,0)
$$

gives indeed the Green's function to the Schrödinger equation for a free particle. Summarizing we have the following theorem:

Theorem 3.1. Let $y \in \mathbb{R}, 0<t<\infty$, then the free Feynman integrand in phase space $I_{0}$ exists as a Hida distribution. Its generating functional $T I_{0}$ is given by (10) and its generalized expectation $\mathbb{E}\left(I_{0}\right)=T I_{0}(0)$ is the Green's function to the Schrödinger equation for the free particle.

3.2. The Feynman-integrand for the harmonic oscillator in phase space. In this section we construct the Feynman integrand for the harmonic oscillator in phase space. For example, the potential is given by $x \mapsto V(x)=\frac{1}{2} k x^{2}, 0 \leq k<\infty$. The corresponding Lagrangian in phase space representation is given by

$$
(x(\tau), p(\tau)) \mapsto L((x(\tau), p(\tau)))=p(\tau) \dot{x}(\tau)-\frac{p(\tau)}{2}-\frac{1}{2} k x(\tau)^{2} .
$$

In addition to the matrix $K$ from the free case (see (8)), we have a matrix $L$ which includes the information about the potential; see also [8]. In order to realize (7) for the 
harmonic oscillator we consider

$$
\begin{aligned}
I_{H O}= & \mathrm{N} \exp \left(-\frac{1}{2}\left\langle\left(\omega_{x}, \omega_{p}\right), K\left(\omega_{x}, \omega_{p}\right)\right\rangle\right) \cdot \exp \left(-\frac{1}{2}\left\langle\left(\omega_{x}, \omega_{p}\right), L\left(\omega_{x}, \omega_{p}\right)\right\rangle\right) \\
& \times \delta\left(\left\langle\left(\omega_{x}, \omega_{p}\right),\left(\mathbb{1}_{[0, t)}, 0\right)\right\rangle-y\right),
\end{aligned}
$$

with

$$
L=\left(\begin{array}{cc}
i k A & 0 \\
0 & 0
\end{array}\right), \quad y \in \mathbb{R}, t>0 .
$$

Here $A f(s)=\mathbb{1}_{[0, t)}(s) \int_{s}^{t} \int_{0}^{\tau} f(r) d r d \tau, f \in L^{2}(\mathbb{R}, \mathbb{C}), s \in \mathbb{R}$. Hence we apply Lemma 2.17 to the case

$$
N=\left(\begin{array}{cc}
\mathbb{1}_{[0, t)^{c}}+i k A & -i \mathbb{1}_{[0, t)} \\
-i \mathbb{1}_{[0, t)} & \mathbb{1}_{[0, t)^{c}}+i \mathbb{1}_{[0, t)}
\end{array}\right)
$$

For determining the inverse of $N$ we use the decomposition of $L_{2}^{2}(\mathbb{R})_{\mathbb{C}}$ into the orthogonal subspaces $L_{2}^{2}([0, t))_{\mathbb{C}}$ and $L_{2}^{2}\left([0, t)^{c}\right)_{\mathbb{C}}$. The operator $N$ leaves both spaces invariant and on $L_{2}^{2}\left([0, t)^{c}\right)$ it is already the identity. Therefore we need just an inversion of $N$ on $L_{2}^{2}([0, t))$. By calculation we obtain

$$
N^{-1}=\left(\begin{array}{cc}
\mathbb{1}_{[0, t)^{c}} & 0 \\
0 & \mathbb{1}_{[0, t)^{c}}
\end{array}\right)-\mathbb{1}_{[0, t)}\left(\begin{array}{cc}
i\left(k A-\mathbb{1}_{[0, t)}\right)^{-1} & i\left(k A-\mathbb{1}_{[0, t)}\right)^{-1} \\
i\left(k A-\mathbb{1}_{[0, t)}\right)^{-1} & i k A\left(k A-\mathbb{1}_{[0, t)}\right)^{-1}
\end{array}\right),
$$

if $\left(k A-\mathbb{1}_{[0, t)}\right)^{-1}$ exists, i.e., $k A-\mathbb{1}_{[0, t)}$ is bijective on $L_{2}^{2}([0, t))$. The operator

$$
k A f(s)=\mathbb{1}_{[0, t)}(s) k \int_{s}^{t} \int_{0}^{\tau} f(r) d r d \tau, \quad f \in L_{2}^{2}([0, t))_{\mathbb{C}}, s \in[0, t),
$$

diagonalizes and the eigenvalues $l_{n}$ that are different from zero have the form:

$$
l_{n}=k\left(\frac{t}{\left(n-\frac{1}{2}\right) \pi}\right)^{2}, \quad n \in \mathbb{N} .
$$

Thus $\left(k A-\mathbb{1}_{[0, t)}\right)^{-1}$ exists if $l_{n} \neq 1$ for all $n \in \mathbb{N}$. For $0<t<\pi /(2 \sqrt{k})$ this is true. The corresponding normalized eigenvectors to $l_{n}$ are

$$
[0, t) \ni s \mapsto e_{n}(s)=\sqrt{\frac{2}{t}} \cos \left(\frac{s}{t}\left(n-\frac{1}{2}\right) \pi\right), \quad s \in[0, t), n \in \mathbb{N} .
$$

Hence we obtain using [7, p. 431, form. 1]:

$$
\begin{aligned}
\frac{1}{\operatorname{det}\left(I d+L(I d+K)^{-1}\right)} & =\operatorname{det}\left(I d+\left(\begin{array}{cc}
-k A & -k A \\
0 & 0
\end{array}\right)\right)^{-1} \\
& =\left(\prod_{n=1}^{\infty}\left(1-k\left(\frac{t}{\left(n-\frac{1}{2}\right) \pi}\right)^{2}\right)\right)^{-1}=\frac{1}{\cos (\sqrt{k} t)}
\end{aligned}
$$

Furthermore, again with $\boldsymbol{\eta}=\left(\mathbb{1}_{[0, t)}, 0\right)$ we obtain

$$
\begin{aligned}
\left(\boldsymbol{\eta}, N^{-1} \boldsymbol{\eta}\right) & =\left(\mathbb{1}_{[0, t)},\left(\mathbb{1}_{[0, t)^{c}}-i\left(\mathbb{1}_{[0, t)}-k A\right)^{-1}\right) \mathbb{1}_{[0, t)}\right)=i \sum_{n=1}^{\infty}\left(1-l_{n}\right)^{-1}\left(\mathbb{1}_{[0, t)}, e_{n}\right)^{2} \\
& =i \sum_{n=1}^{\infty} \frac{1}{1-k\left(\frac{t}{\left(n-\frac{1}{2}\right) \pi}\right)^{2}} \frac{2 t}{\left(\left(n-\frac{1}{2}\right) \pi\right)^{2}}=2 i t \sum_{n=1}^{\infty} \frac{1}{\left(\left(n-\frac{1}{2}\right) \pi\right)^{2}-k t^{2}} \\
& =\frac{i}{\sqrt{k}} 8 \sqrt{k} t \sum_{n=1}^{\infty} \frac{1}{((2 n-1) \pi)^{2}-4 k t^{2}}=\frac{i}{\sqrt{k}} \tan (\sqrt{k} t)=i \frac{\tan (\sqrt{k} t)}{\sqrt{k}},
\end{aligned}
$$


by using [7, p. 421, form. 1]. Hence we have for the $T$-transform in $\mathbf{f} \in S_{2}(\mathbb{R})$ by applying Lemma 2.17

$$
\begin{aligned}
T I_{H O}(\mathbf{f})= & \sqrt{\left(\frac{\sqrt{k}}{2 \pi i \sin (\sqrt{k} t)}\right)} \exp \left(-\frac{1}{2} \frac{\sqrt{k}}{i \tan (\sqrt{k} t)}(y-(\boldsymbol{\eta}, \mathbf{f}+\mathbf{g}))^{2}\right) \\
& \times \exp \left(-\frac{1}{2}\left((\mathbf{f}+\mathbf{g}),\left(\begin{array}{cc}
\mathbb{1}_{[0, t)^{c}} & 0 \\
\mathbb{1}_{[0, t)^{c}}
\end{array}\right)(\mathbf{f}+\mathbf{g})\right)\right) \\
& \times \exp \left(-\frac{1}{2}((\mathbf{f}+\mathbf{g}), Q(k, t)(\mathbf{f}+\mathbf{g}))\right),
\end{aligned}
$$

where

$$
Q(k, t)=\left(\begin{array}{cc}
-i \mathbb{1}_{[0, t)}\left(k A-\mathbb{1}_{[0, t)}\right)^{-1} & -i \mathbb{1}_{[0, t)}\left(k A-\mathbb{1}_{[0, t)}\right)^{-1} \\
-i \mathbb{1}_{[0, t)}\left(k A-\mathbb{1}_{[0, t)}\right)^{-1} & -i k \mathbb{1}_{[0, t)} A\left(k A-\mathbb{1}_{[0, t)}\right)^{-1}
\end{array}\right) .
$$

Summarizing we have the following theorem:

Theorem 3.2. Let $y \in \mathbb{R}, 0<t<\pi /(2 \sqrt{k})$, then the Feynman integrand for the harmonic oscillator in phase space $I_{H 0}$ exists as a Hida distribution and its generating functional is given by (11). Moreover, its generalized expectation

$$
\mathbb{E}\left(I_{H O}\right)=T\left(I_{H O}\right)(0)=\sqrt{\left(\frac{\sqrt{k}}{2 \pi i \sin (\sqrt{k} t)}\right)} \exp \left(i \frac{\sqrt{k}}{2 \tan (\sqrt{k} t)} y^{2}\right)
$$

is the Green's function to the Schrödinger equation for the harmonic oscillator, compare, e.g., with [15].

\section{Canonical commutation relations}

In this section we give a functional form of the quantum mechanical commutator relations. The definition can be found in [6], for their realization in the white noise framework, we refer to [25, Chap. 9]. With the help of these relations we can confirm that the choice of the phase space variables, as in (3), gives the right physics. For example, the variables fulfill the noncommutativity of momentum and position variables at equal times. This seemed to have no direct translation in a path integral formulation of quantum mechanics. But on a heuristic level Feynman and Hibbs [6] found an argument to show that $\mathbb{E}\left(p(t+\varepsilon) x(t) I_{V}\right) \neq \mathbb{E}\left(p(t-\varepsilon) x(t) I_{V}\right)$ for infinitesimal small $\varepsilon$ and that the difference is given by the commutator. First we collect some helpful formulas.

Lemma 4.1. Let $\Phi \in(S)^{\prime}, \mathbf{k} \in S_{d}(\mathbb{R})$ and $n \in \mathbb{N}$, then

$$
\left.(-i)^{n} \frac{d^{n}}{d \lambda^{n}} T \Phi(\lambda \mathbf{k}+\mathbf{f})\right|_{\lambda=0}=T\left(\langle\mathbf{k}, \cdot\rangle^{n} \cdot \Phi\right)(\mathbf{f}), \quad \mathbf{f} \in S_{d}(\mathbb{R}) .
$$

The proof of this lemma is an easy application of Corollary 2.5. Note that for $\Phi \in(S)^{\prime}$, $\mathbf{k} \in S_{d}(\mathbb{R}), n \in \mathbb{N}$ the product $\langle\mathbf{k}, \cdot\rangle^{n} \cdot \Phi$ in Lemma 4.1 is defined by using that $(S)$ is a continuous algebra w.r.t. the pointwise product. In the following for $\boldsymbol{\eta}_{i}, \mathbf{k} \in L_{d}^{2}(\mathbb{R})$ and $y_{i} \in \mathbb{R}, i \in(1, \ldots, J)$, we use the abbreviations:

$$
\left\langle\boldsymbol{\eta}, N^{-1} \mathbf{k}\right\rangle=\left(\left(\boldsymbol{\eta}_{1}, N^{-1} \mathbf{k}\right), \ldots,\left(\boldsymbol{\eta}_{J}, N^{-1} \mathbf{k}\right)\right) \in \mathbb{R}^{J}
$$

and $y=\left(y_{1}, \ldots, y_{J}\right) \in \mathbb{R}^{J}$. 
Proposition 4.2. Let $\Phi_{K, L}$ be as in Lemma 2.17. Then for $\mathbf{k}, \mathbf{h} \in L_{2}^{2}(\mathbb{R})$,

$$
\langle\mathbf{k}, \cdot\rangle \cdot \Phi_{K, L}
$$

and

$$
\langle\mathbf{h}, \cdot\rangle \cdot\langle\mathbf{k}, \cdot\rangle \cdot \Phi_{K, L}
$$

exist as Hida distributions. Furthermore, for $\mathbf{f} \in S_{d}(\mathbb{R})$,

$$
\begin{aligned}
& T\left(\langle\mathbf{k}, \cdot\rangle \cdot \Phi_{K, L}\right)(\mathbf{f}) \\
& \quad=i T \Phi_{K, L}(\mathbf{f})\left(\left(\mathbf{f}, N^{-1} \mathbf{k}\right)+\left(\left\langle\boldsymbol{\eta}, N^{-1} \mathbf{k}\right\rangle, M_{N^{-1}}^{-1}\left(i y+\left\langle\boldsymbol{\eta}, N^{-1} \mathbf{f}+\mathbf{g}\right\rangle\right)\right)\right)
\end{aligned}
$$

and

$$
\begin{aligned}
T\left(\langle\mathbf{k}, \cdot\rangle \cdot\langle\mathbf{h}, \cdot\rangle \cdot \Phi_{K, L}\right)(\mathbf{f}) \\
=T\left(\Phi_{K, L}\right)(\mathbf{f}) \\
\quad \times\left(\left(\left(\mathbf{k}, N^{-1} \mathbf{h}\right)+\left(\left\langle\boldsymbol{\eta}, N^{-1} \mathbf{h}\right\rangle, M_{N^{-1}}^{-1}\left\langle\boldsymbol{\eta}, N^{-1} \mathbf{k}\right\rangle\right)\right)\right. \\
\quad-\left(\left((\mathbf{f}+\mathbf{g}), N^{-1} \mathbf{h}\right)+\left(\left(i y+\left\langle\boldsymbol{\eta}, N^{-1} \mathbf{f}+\mathbf{g}\right\rangle\right), M_{N^{-1}}^{-1}\left\langle\boldsymbol{\eta}, N^{-1} \mathbf{h}\right\rangle\right)\right) \\
\left.\quad \times\left(\left((\mathbf{f}+\mathbf{g}), N^{-1} \mathbf{k}\right)+\left(\left(i y+\left\langle\boldsymbol{\eta}, N^{-1} \mathbf{f}+\mathbf{g}\right\rangle\right), M_{N^{-1}}^{-1}\left\langle\boldsymbol{\eta}, N^{-1} \mathbf{k}\right\rangle\right)\right)\right) .
\end{aligned}
$$

Proof. We have from Lemma 4.1 that

$$
T\left(\langle\mathbf{k}, \cdot\rangle \cdot \Phi_{K, L}\right)(\mathbf{f})=\left.\frac{1}{i} \frac{d}{d \lambda} T\left(\Phi_{K, L}\right)(\mathbf{f}+\lambda \mathbf{k})\right|_{\lambda=0}, \quad \mathbf{k} \in S_{d}(\mathbb{R}) .
$$

Then by Lemma 2.17,

$$
\begin{aligned}
T\left(\Phi_{K, L}\right)(\mathbf{f}+\lambda \mathbf{k})= & T\left(\Phi_{K, L}\right)(\mathbf{f}) \exp \left(-\frac{1}{2} \lambda^{2}\left(\mathbf{k}, N^{-1} \mathbf{k}\right)-\lambda\left(\mathbf{f}, N^{-1} \mathbf{k}\right)\right) \\
& \times \exp \left(-\frac{1}{2} \lambda^{2}\left(\left\langle\boldsymbol{\eta}, N^{-1} \mathbf{k}\right\rangle, M_{N^{-1}}^{-1}\left\langle\boldsymbol{\eta}, N^{-1} \mathbf{k}\right\rangle\right)\right. \\
& \left.-\lambda\left(\left\langle\boldsymbol{\eta}, N^{-1} \mathbf{k}\right\rangle, M_{N^{-1}}^{-1}\left(i y+\left\langle\boldsymbol{\eta}, N^{-1} \mathbf{f}+\mathbf{g}\right\rangle\right)\right)\right) .
\end{aligned}
$$

Thus, by the above formula we get

$$
\begin{aligned}
\frac{1}{i} \frac{d}{d \lambda} T & \left(\Phi_{K, L}\right)(\mathbf{f}+\lambda \mathbf{k}) \\
= & -i T \Phi_{K, L}(\mathbf{f}) \\
& \times\left(-\left(\left(\mathbf{f}, N^{-1} \mathbf{k}\right)+\left(\left\langle\boldsymbol{\eta}, N^{-1} \mathbf{k}\right\rangle, M_{N^{-1}}^{-1}\left(i y+\left\langle\boldsymbol{\eta}, N^{-1} \mathbf{f}+\mathbf{g}\right\rangle\right)\right)\right)\right. \\
& \left.-\lambda\left(\left(\mathbf{k}, N^{-1} \mathbf{k}\right)+\left(\left\langle\boldsymbol{\eta}, N^{-1} \mathbf{k}\right\rangle, M_{N^{-1}}^{-1}\left\langle\boldsymbol{\eta}, N^{-1} \mathbf{k}\right\rangle\right)\right)\right) .
\end{aligned}
$$

Then by an approximation in the sense of Corollary 2.5 we get $\langle\mathbf{k}, \cdot\rangle \cdot \Phi_{K, L} \in(S)^{\prime}$ for $\mathbf{k} \in L_{d}^{2}(\mathbb{R})$. Setting $\lambda=0$ we obtain the desired expression. In an analogous way one can show the second formula by using the second derivative; see Lemma 4.1 and the polarization identity.

Next we extend this to the case, where just one of the functions is in $L_{2}^{2}(\mathbb{R})$, but the other one is a tempered distribution. 
Definition 4.3. Let $\mathbf{h} \in L_{d}^{2}(\mathbb{R})$ and $\mathbf{k} \in S_{d}^{\prime}(\mathbb{R})$ with compact support and let $\left(\psi_{n}\right)_{n \in \mathbb{N}}$ be a standard approximate identity. Since the convolution of a compactly supported smooth function with a compactly supported tempered distribution gives a Schwartz test function, i.e., $\psi_{n} * \mathbf{k} \in S_{d}(\mathbb{R}), n \in \mathbb{N}$ (see e.g. [24, Chap. 9]) we may define

$$
\langle\mathbf{k}, \cdot\rangle \cdot\langle\mathbf{h}, \cdot\rangle \cdot \Phi_{K, L}:=\lim _{n \rightarrow \infty}\left\langle\psi_{n} * \mathbf{k}, \cdot\right\rangle \cdot\langle\mathbf{h}, \cdot\rangle \cdot \Phi_{K, L},
$$

in this case the limit exists in the sense of Corollary 2.5.

In the following for convenience we restrict ourselves to the case $d=2$. For the free Feynman integrand we then have as an analogue to [25]:

Theorem 4.4. Let $0<s-\varepsilon<s<s+\varepsilon<t<\infty$, then

$$
\left\langle\left(0, \delta_{s \pm \varepsilon}\right), \cdot\right\rangle \cdot\left\langle\left(\mathbb{1}_{[0, s)}, 0\right), \cdot\right\rangle \cdot I_{0} \in(S)^{\prime}
$$

and

$$
\lim _{\varepsilon \rightarrow 0}\left(T\left(\left\langle\delta_{s+\varepsilon}, \cdot\right\rangle \cdot\left\langle\mathbb{1}_{[0, s)}, \cdot\right\rangle \cdot I_{0}\right)(\mathbf{0})-T\left(\left\langle\delta_{s-\varepsilon}, \cdot\right\rangle \cdot\left\langle\mathbb{1}_{[0, s)}, \cdot\right\rangle \cdot I_{0}\right)(\mathbf{0})\right)=-i T\left(I_{0}\right)(\mathbf{0}) .
$$

Proof. Set $\boldsymbol{\psi}_{n}^{ \pm}:=\psi_{n} *\left(0, \delta_{s \pm \varepsilon}\right), n \in \mathbb{N}$, where $\left(\psi_{n}\right)_{n \in \mathbb{N}}$ is a standard approximate identity. Note that $\lim _{n \rightarrow \infty}\left\langle\boldsymbol{\psi}_{n}^{ \pm},\left(0, \mathbb{1}_{[0, s)}\right)\right\rangle=\frac{1}{2} \pm \frac{1}{2}$. Using (10) in the case $\boldsymbol{\eta}=\left(\mathbb{1}_{[0, t)}, 0\right)$ with

$$
N^{-1}=\left(\begin{array}{cc}
\mathbb{1}_{[0, t)^{c}}+i \mathbb{1}_{[0, t)} & i \mathbb{1}_{[0, t)} \\
i \mathbb{1}_{[0, t)} & \mathbb{1}_{[0, t)^{c}}
\end{array}\right)
$$

as in (9), we have $\left(M_{N^{-1}}\right)^{-1}=(i t)^{-1}$. Thus, together with Proposition 4.2 we obtain

$$
\begin{aligned}
& T\left(\left\langle\boldsymbol{\psi}_{n}^{ \pm}, \cdot\right\rangle \cdot\left\langle\left(\mathbb{1}_{[0, s)}, 0\right), \cdot\right\rangle \cdot I_{0}\right)(\mathbf{f}) \\
&=T\left(I_{0}\right)(\mathbf{f})(\left(\left(\Psi_{n}^{ \pm}, O(s)+\left(\left(O(t),\left(\mathbb{1}_{[0, s)}, 0\right)\right), \frac{1}{i t}\left(O(t), \Psi_{n}^{ \pm}\right)\right)\right)\right) \\
&-\left(\left((\mathbf{f}, O(s))+\left((i y+O(t), \mathbf{f}), \frac{1}{i t}\left(O(t),\left(\mathbb{1}_{[0, s)}, 0\right)\right)\right)\right)\right. \\
&\left.\left.\times\left(\left(N^{-1} \mathbf{f}, \Psi_{n}^{ \pm}\right)+\left((i y+(O(t), \mathbf{f})), \frac{1}{i t}\left(O(t), \Psi_{n}^{ \pm}\right)\right)\right)\right)\right),
\end{aligned}
$$

where

$$
O(x)=\left(i \mathbb{1}_{[0, x)}, i \mathbb{1}_{[0, x)}\right) .
$$

Now let us take a look at the terms which include the sequence $\Psi_{n}^{ \pm}$.

Since $N^{-1}$ consists of projections on $[0, t)$ or $[0, t)^{c}$, respectively, and $\int_{\mathbb{R}} \Psi_{n}^{ \pm}(s) d s=1$, we have $\left|\left(N^{-1} \mathbf{f}, \Psi_{n}^{ \pm}\right)\right| \leq\|\mathbf{f}\|_{\text {sup }}$. Furthermore, $\left|\left(\left(i \mathbb{1}_{[0, u)}, i \mathbb{1}_{[0, u)}\right), \Psi_{n}^{ \pm}\right)\right| \leq 1$, for all $n \in \mathbb{N}$ and $0<u \leq t$. Therefore, the expression can be bounded uniformly in $n \in \mathbb{N}$ in the sense of Corollary 2.5 (note that $\|\cdot\|_{\text {sup }} \leq\|\cdot\|_{p}$ for some $p \in \mathbb{N}$ ). Obviously the $T$ transform in (12) is convergent as $n \rightarrow \infty$, thus the limit exists as a Hida distribution by Corollary 2.5. Taking the limit leads us to

$$
\begin{gathered}
T\left(I_{0}\right)(\mathbf{f})\left(\frac{s}{i t}+i \mathbb{1}_{[0, s)}(s \pm \varepsilon)-\left(\frac{i}{t}\left(s \cdot\left(y+\int_{[0, t)} f_{x}+f_{p} d s\right)\right)-\left(i \int_{0}^{s} f_{x}+f_{p} d t\right)\right)\right. \\
\left.\times\left(\frac{i}{t}\left(y+\int_{[0, t)} f_{x}+f_{p} d s\right)-\left(f_{x}(s \pm \varepsilon)\right)\right)\right), \\
\mathbf{f}=\left(f_{x}, f_{p}\right) \in S_{2}(\mathbb{R}) .
\end{gathered}
$$


For the difference $\mathbb{E}\left(\left\langle\delta_{s+\varepsilon}, \cdot\right\rangle\left\langle\mathbb{1}_{[0, s)}, \cdot\right\rangle I_{0}\right)-\mathbb{E}\left(\left\langle\delta_{s-\varepsilon}, \cdot\right\rangle\left\langle\mathbb{1}_{[0, s)}, \cdot\right\rangle I_{0}\right)$ we have

$$
\begin{aligned}
\lim _{\varepsilon \rightarrow 0} T & \left(\left\langle\delta_{s+\varepsilon}, \cdot\right\rangle\left\langle\mathbb{1}_{[0, s)}, \cdot\right\rangle I_{0}\right)(\mathbf{0})-T\left(\left\langle\delta_{s-\varepsilon}, \cdot\right\rangle\left\langle\mathbb{1}_{[0, s)}, \cdot\right\rangle I_{0}\right)(\mathbf{0}) \\
\quad & \lim _{\varepsilon \rightarrow 0} T\left(I_{0}\right)(\mathbf{0})\left(i \mathbb{1}_{[0, s)}(s+\varepsilon)-i \mathbb{1}_{[0, s)}(s-\varepsilon)\right)=T\left(I_{0}\right)(\mathbf{0}) \cdot(0-i)=-i T\left(I_{0}\right)(\mathbf{0}),
\end{aligned}
$$

which completes the proof.

Thus, the commutation law for the free Feynman integrand in phase space is fulfilled in the sense of Feynman and Hibbs [6].

\section{BIBLIOGRAPHY}

1. S. Albeverio, G. Guatteri, and S. Mazzucchi, Phase space Feynman path integrals, J. Math. Phys. 43 (2002), no. 6, 2847. MR1902454 (2003f:81148)

2. S. Albeverio, R. Høegh-Krohn, and S. Mazzucchi, Mathematical Theory of Feynman Path Integrals: An Introduction, Lecture Notes in Mathematics, vol. 523, Springer-Verlag, BerlinHeidelberg-New York, 2008. MR2453734 (2010e:58008)

3. Y. M. Berezansky and Y. G. Kondratiev, Spectral Methods in Infinite-Dimensional Analysis, vol. 2, Kluwer Academic Publishers, Dordrecht, 1995. MR.1340627 (96d:46001b)

4. R. P. Feynman, Space-time approach to non-relativistic quantum mechanics, Reviews of Modern Physics 20 (1948), 367-387. MR0026940 (10:224b)

5. R. P. Feynman, An operator calculus having applications in quantum electrodynamics, Physical Review 84 (1951), no. 1, 108-124. MR0044379 (13:410e)

6. R. P. Feynman and A. R. Hibbs, Quantum Mechanics and Path Integrals, McGraw-Hill, London-New York, 1965. MR197789

7. I. S. Gradshteyn and I. M. Ryzhik, Table of Integrals, Series, and Products, Academic Press, New York, 1965.

8. M. Grothaus and L. Streit, Quadratic actions, semi-classical approximation, and delta sequences in Gaussian analysis, Rep. Math. Phys. 44 (1999), no. 3, 381-405. MR1737385 (2001b:81064)

9. I. M. Gel'fand and N. Ya. Vilenkin, Generalized Functions, vol. 4, Academic Press, New YorkLondon, 1968. MR0435834 (55:8786d)

10. T. Hida, Brownian Motion, Springer-Verlag, New York, 1980. MR562914 (81a:60089)

11. T. Hida, H.-H. Kuo, J. Potthoff, and L. Streit, White Noise. An Infinite Dimensional Calculus, Kluwer Academic Publisher, Dordrecht-Boston-London, 1993. MR.1244577 (95f:60046)

12. T. Hida and L. Streit, Generalized Brownian functionals and the Feynman integral, Stoch. Proc. Appl. 16 (1983), 55-69. MR723643 (86d:60048)

13. J. R. Klauder and I. Daubechies, Measures for path integrals, Physical Review Letters 48 (1982), no. 3, 117-120. MR639863 (83c:81031)

14. J. R. Klauder and I. Daubechies, Quantum mechanical path integrals with Wiener measures for all polynomial Hamiltonians, Physical Review Letters 52 (1984), no. 14, 1161-1164. MR736821 (85b:81068)

15. D. C. Khandekar and S. V. Lawande, Feynman path integrals: Some exact results and applications, Physics Reports 137 (1985), no. 2-3, 115-229. MR839624(87i:81077)

16. Yu. G. Kondratiev, P. Leukert, J. Potthoff, L. Streit, and W. Westerkamp, Generalized functionals in Gaussian spaces: The characterization theorem revisited, J. Funct. Anal. 141 (1996), no. 2, 301-318 MR1418508 (97j:60070)

17. Yu. G. Kondratiev, Spaces of entire functions of an infinite number of variables, connected with the rigging of a Fock space, Selecta Mathematica Sovietica 10 (1991), no. 2, 165-180. MR 1115043

18. H.-H. Kuo, White Noise Distribution Theory, CRC Press, Boca Raton-New York-LondonTokyo, 1996. MR:1387829 (97m:60056)

19. A. Lascheck, P. Leukert, L. Streit, and W. Westerkamp, More about Donsker's delta function, Soochow Journal of Mathematics 20 (1994), no. 3, 401-418. MR1292245 (95k:60100)

20. R. A. Minlos, Generalized random processes and their extension to a measure, Selected Transl. Math. Statist. and Prob. 3 (1963), 291-313. MR0154317 (27:4266)

21. N. Obata, White Noise Calculus and Fock Spaces, Springer-Verlag, Berlin-Heidelberg-New York, 1994. MR1301775 (96e:60061)

22. J. Potthoff and L. Streit, A characterization of Hida distributions, J. Funct. Anal. 101 (1991), 212-229. MR 1132316 (93a:46078) 
23. M. Reed and B. Simon, Methods of Modern Mathematical Physics, vol. I, Academic Press, New York-London, 1975. MR0493420 (58:12429b)

24. M. Reed and B. Simon, Methods of Modern Mathematical Physics, vol. II, Academic Press, New York-London, 1975. MR0493420 (58:12429b)

25. W. Westerkamp, Recent Results in Infinite Dimensional Analysis and Applications to Feynman Integrals, PhD Thesis, University of Bielefeld, 1995.

Functional Analysis and Stochastic Analysis Group, Department of Mathematics, UniverSity of Kaiserslautern, 67653 Kaiserslautern, Germany

E-mail address: bock@mathematik.uni-kl.de

Functional Analysis and Stochastic Analysis Group, Department of Mathematics, UniverSity of Kaiserslautern, 67653 Kaiserslautern, Germany

E-mail address: grothaus@mathematik.uni-kl.de

Received 03/DEC/2010

Originally published in English 